\title{
A Model of Endogenous Growth: The Case of an Innovative Economy
}

\author{
Constantin Chilarescu ${ }^{1}$ and Ioana Viasu ${ }^{2}$ \\ ${ }^{1}$ Laboratory CLERSE, University of Lille 1, Cité Scientifique, 59655 Villeneuve d'Ascq Cedex, France \\ ${ }^{2}$ Faculty of Economics and Business Administration, West University of Timisoara, Strada J. H. Pestalozzi, \\ Nr. 16, 300115 Timisoara, Romania
}

Correspondence should be addressed to Constantin Chilarescu; constantin.chilarescu@univ-lillel.fr

Received 5 August 2013; Accepted 14 November 2013

Academic Editors: D. M. Hanink, B. Junquera, Y. Shiftan, and J. Zarnikau

Copyright (C) 2013 C. Chilarescu and I. Viasu. This is an open access article distributed under the Creative Commons Attribution License, which permits unrestricted use, distribution, and reproduction in any medium, provided the original work is properly cited.

The main aim of this paper is to bring some improvements to the model developed by Funke and Strulik, having as starting point the basic model proposed by Grossman and Helpman. We will prove that the competitive equilibrium solution is locally unique. Nevertheless, at least as regards the stability of equilibrium point, we confirm the results obtained by Funke and Strulik.

\section{Introduction}

A large number of papers have been published in the last years on this subject, following the line developed by Grossman and Helpman [1]. Among them, to our knowledge, the first are the papers of Romer [2] and Eriksson [3]. Eriksson works out a model that is only a slight modification to those developed by Grossman and Helpman. The formal model of the economy of Romer has three sectors. The research sector uses human capital and the existing stock of knowledge to produce new knowledge. The intermediate-goods sector uses the designs from the research sector together with forgone output to produce the large number of intermediates that are in fact durable goods that are available for use in final goods production at any time. The final goods sector uses labor, human capital, and the set of intermediates that are available to produce final output. Output can be either consumed or saved as new capital.

Few years later, the balanced growth path of the endogenous growth model with physical capital, human capital, and R\&D has been explored by Funke and Strulik [4] and then by Arnold [5] (henceforth FSA). FSA suggest that the typical advanced economy follows three development phases, characterized by physical capital accumulation, human capital formation, and innovation. In their paper, FSA claim that consumption goods, investment goods, and intermediate goods are all produced with the same technology and they can be transformed one to one without further cost from output of the industrial sector which is produced with Cobb-Douglas technology. In other words, one unit of each intermediate can be obtained from one unit of final output.

Gómez [6] analyzes the equilibrium dynamics of this model and corrects the analysis of FSA. Sequeira [7] incorporates an erosion effect into the endogenous growth model and claims that this effect significantly improves the fit between the model and the data.

Iacopetta $[8,9]$ extends the earlier analysis of FSA and argues that other sequences of the phases of development are possible and shows that the model can generate a trajectory in which innovation precedes human capital formation. This trajectory accords with the observation that the rise in formal education is followed with a considerable lag in the process of industrialization.

In order to obtain our results, we follow the line developed by Grossman and Helpman, more exactly, the model proposed in Chapter 5 . They consider the case of an economy where capital goods and intermediate inputs each have only a single use and the final output is either consumed or invested. In their model, labor is used in $\mathrm{R} \& \mathrm{D}$, in the production of intermediates and final goods. 
We concentrate our analysis only on the case of an innovative economy and the paper is organized as follows. In the next section we argue and develop the differential equations that describe the dynamics of all variables of the model. The third section gives a complete characterization of the balanced growth path. Section four studies the stability conditions and the final section presents some conclusions and numerical simulations.

\section{A Model of Growth with Innovation}

In this section we develop a model of endogenous growth with innovation and derive the differential equations that describe the dynamics of the economy. Without loss of generality, we suppose that the economy is populated by a large and constant number of identical individuals, normalized to one, so that all the variables can be interpreted as per capita quantities. Each individual is endowed with one unit of time. There are three types of sectors in this model: the final output sector, the R\&D sector, and the educational sector. Without loss of generality we introduce the following assumptions.

(a) The first sector produces the final output and the technology requires inputs, besides intermediates, labor, and physical capital. Capital is measured in units of consumption goods. Output can be either consumed or saved as new capital. The market for the final good $y$ is assumed to be perfectly competitive and therefore the price of this good equals its marginal production cost. Final output $y$ is produced according to a Cobb-Douglas production function

$$
y=\gamma k^{\beta} d^{\eta} h_{y}^{1-\beta-\eta}
$$

where $\gamma>0, \beta$, and $\eta$ are positive elasticity parameters with $\beta+\eta \leq 1, k$ is physical capital, $h_{y}$ denotes the share of human capital employed to produce the final output, and $d$ is an index of intermediate goods,

$$
d=\left(\int_{0}^{n} x(i)^{\alpha} d i\right)^{1 / \alpha}
$$

where $n=n(t)$ is a measure of products invented before time $t$ and can be considered as the number of available varieties. $x(i)$ represents the input of component $i$ in the production of final good, and $0<$ $\alpha<1$ controls the elasticity of substitution between intermediates, $\varepsilon=1 /(1-\alpha)$.

(b) In the second sector, specialized R\&D firms devote resources to invent new products. Invention of new products is determined solely by the share of human capital devoted to research activity, denoted here by $h_{d}$. The measure of products invented evolves according to the following differential equation:

$$
\dot{n}=\delta h_{d},
$$

where $\delta>0$ is an efficiency parameter. (c) Once a product was invented, a R\&D firm obtains a perpetual patent, which allows the firm to sell these new products to the final good sector at a price chosen to maximize its profit. Whether the owner of the patent manufactures itself the good or licenses others to do so, it can extract the same monopoly profit. Intermediate goods are produced by labor alone, under monopolistic competition, and therefore, in order to fulfill the conditions for market clearing, we need to consider this kind of labor in the model. In fact intermediate goods are durable goods used by the final output sector. Since the intermediates are assumed not to depreciate, the value of one unit of it is the present discounted value of the infinite stream of rental income that it generates.

(d) The third sector is the educational sector. Each individual can spend a part of his time in the education sector, in order to raise its level of human capital. This process is proportional to the share of human capital devoted to education, denoted here by $h_{e}$ and is described by the following differential equation:

$$
\dot{h}=\xi h_{e}
$$

where $\xi>0$ is an efficiency parameter.

Under these assumptions we are now able to determine the monopolist price of each intermediates. The derivative of function $d$, defined by (2), with respect to $x(j)$, is given by

$$
\frac{d}{d x(j)}[d]=d^{1-\alpha} x(j)^{\alpha-1},
$$

and therefore

$$
p(j)^{d}=d^{1-\alpha} x(j)^{\alpha-1} p_{d}
$$

is the price of intermediate $x(j)$ in terms of $d$, where $p_{d}$ is the unit price of $d$. By assuming in a formal way that the marginal and average cost of production is constant, normalized to one, the lemma of Shephard provides the following prices, in terms of $y$

$$
p_{d}=\eta \frac{y}{d}, \quad r=\beta \frac{y}{k}, \quad w=(1-\beta-\eta) \frac{y}{h_{y}},
$$

where $w$ is the price of labor employed for the production of capital good. In the same terms, substituting $p_{d}$ from (7) into (6), the price of any intermediate $x(j)$, denoted here by $p(j)$, will then be given by

$$
p(j)=\frac{\eta y}{d^{\alpha}} x(j)^{\alpha-1}
$$

This price is paid by the final output sector, for a unit of $x(j)$, to monopolist producer. As we can observe, this price is dependent on $x(j)$. Therefore, the demand function for an intermediate $x(j)$ at price $p(j)$ will be

$$
x(j)=\left[\frac{d^{\alpha}}{\eta y}\right]^{1 /(\alpha-1)} p(j)^{1 /(\alpha-1)} .
$$


Facing the demand function (9), the monopolist supplier of intermediate $x_{j}$ maximizes operating profits

$$
\pi(j)=(p(j)-w) x(j) .
$$

The optimal price will therefore be given by

$$
p_{j}=\frac{w}{\alpha}
$$

To simplify, we denote by $p_{x}$ this unique price. In a symmetric equilibrium, the quantity supplied for all intermediates is the same; that is, $x(j)=x$, and therefore

$$
d^{\alpha}=n x^{\alpha}
$$

Substituting this result into (8) yields

$$
p_{x}=\frac{\eta y}{n x}
$$

Equating the two prices yields the total quantity of intermediates required by the sector of production:

$$
n x=\frac{\alpha \eta}{1-\beta-\eta} h_{y} .
$$

Regardless of its composition, $X=n x$ measures the quantity of labor used in manufacturing intermediates. We denote by $h_{m}$ this quantity of labor; that is,

$$
h_{m}=\frac{\alpha \eta}{1-\beta-\eta} h_{y}
$$

Let $\left(u_{d}, u_{m}, u_{e}, u_{y}\right)$ be the amount of unit time necessary to innovation, to manufacture intermediates, to education and, respectively, to produce the final output. Then, we have, $h_{y}=$ $h u_{y}, h_{d}=h u_{d}, h_{m}=h u_{m}$, and $h_{e}=h u_{e}$ and the full employment requires:

$$
u_{y}+u_{d}+u_{m}+u_{e}=1
$$

Under this hypothesis, (15) can therefore be written as

$$
u_{m}=\frac{\alpha \eta}{1-\beta-\eta} u_{y}
$$

and the above identity becomes

$$
\frac{1-\beta-\eta(1-\alpha)}{1-\beta-\eta} u_{y}+u_{d}+u_{e}=1 .
$$

Substitution of $d$ from (12) and $n x$ from (14) into (1) provides the final form of production function:

$$
y=A k^{\beta} h_{y}^{1-\beta} n^{\eta(1-\alpha) / \alpha}, \quad A=\gamma\left(\frac{\alpha \eta}{1-\beta-\eta}\right)^{\eta} .
$$

Successively substitution of $w$ from (7), $p_{j}$ from (11), and $x$ from (14) into (10) provides a profit equal to

$$
\pi=\frac{\eta(1-\alpha)}{n} y \text {. }
$$

If $v$ is the market price of an intermediate, then, in a general equilibrium, free entry into $\mathrm{R} \& \mathrm{D}$ requires

$$
w=\delta v \Longleftrightarrow g_{v}=\frac{\dot{v}}{v}=\frac{\dot{w}}{w}=g_{w}
$$

where $g_{z}$ denotes the growth rate of variable $z$. The equilibrium in the capital market requires that the interest rate equals the dividend rate $\pi / \nu$ plus the rate of capital gain $\dot{\nu} / \nu$ and thus we can write

$$
g_{v}=\frac{\dot{v}}{v}=r-\frac{\pi}{v}=g_{w} .
$$

Substituting $\pi$ from (20), $\nu$ from (21) and $w$ from (7) we obtain

$$
g_{w}=r-\frac{\delta \eta(1-\alpha)}{1-\beta-\eta} \frac{h_{y}}{n} .
$$

The next two equations describe the dynamics of the budget constraint and the dynamics of physical capital

$$
\begin{gathered}
\dot{a}=r a+w\left(1-u_{e}\right) h-c, \\
\dot{k}=y-c .
\end{gathered}
$$

Subject to the budget constraint equation (24) and to the development of skill equation (4) and using as state variables $(h, a)$ and as control variables $\left(c, u_{e}\right)$, we can write the following optimization problem:

$$
\max _{\left\{c, u_{e}\right\}} \int_{0}^{\infty} \frac{c^{1-\theta}-1}{1-\theta} e^{-\rho t} d t
$$

where $\rho>0$ denotes the time preference rate and $\theta>0$ denotes the intertemporal elasticity of substitution. The Hamiltonian is given by

$$
J=\frac{c^{1-\theta}-1}{1-\theta}+\lambda\left[r a+w\left(1-u_{e}\right) h-c\right]+\mu \xi h u_{e} .
$$

The first derivatives of Hamiltonian with respect to $c, u_{e}, h$, and $a$ provide the following system of differential equations:

$$
\begin{aligned}
& g_{c}=\frac{\dot{c}}{c}=\frac{r-\rho}{\theta}, \\
& g_{w}=\frac{\dot{w}}{w}=r-\xi,
\end{aligned}
$$

where $g_{z}$ denotes the growth rate of variable $z$. Combining (23) with the second equation of the above system we can determine the following relation

$$
u_{y}=\frac{\xi(1-\beta-\eta)}{\delta \eta(1-\alpha)} \frac{n}{h}
$$

from where it immediately follows

$$
g_{u_{y}}=g_{n}-g_{h} \text {. }
$$


After log-differentiating equation (19) with respect to time, the output growth rate is then determined by

$$
g_{y}=\beta g_{k}+(1-\beta)\left(g_{h}+g_{u_{y}}\right)+\frac{\eta(1-\alpha)}{\alpha} g_{n},
$$

and substitution of the above result provides

$$
g_{y}=\beta g_{k}+\frac{\alpha(1-\beta)+\eta(1-\alpha)}{\alpha} g_{n} .
$$

Equations (3) and (25) can also be written as

$$
\begin{gathered}
g_{n}=\delta \frac{h}{n} u_{d}, \\
g_{k}=\frac{1}{\beta} r-\frac{c}{k} .
\end{gathered}
$$

Log-differentiation of $r$ and $w$ from (7) provides

$$
\begin{gathered}
g_{r}=-\frac{1-\beta}{\beta} r+(1-\beta) \frac{c}{k}+\frac{\alpha(1-\beta)+\eta(1-\alpha)}{\alpha} g_{n} \\
g_{w}=g_{y}-\left(g_{h}+g_{u_{y}}\right)=g_{y}-g_{n} \Longrightarrow g_{n}=g_{y}-g_{w}
\end{gathered}
$$

and after some algebraic manipulations yields

$$
g_{n}=\frac{\alpha}{\eta(1-\alpha)-\alpha \beta}\left(\beta \frac{c}{k}-\xi\right) .
$$

Substituting (36) into (34) and denoting by $\chi=c / k$ and $\psi=n / h$ enable us to write down the following system of differential equations in terms of $(r, \chi, \psi)$ :

$$
\begin{gathered}
g_{r}=-\frac{(1-\beta)}{\beta} r+\frac{\eta(1-\alpha)}{\eta(1-\alpha)-\alpha \beta} \chi-A, \\
A=\frac{\xi[\alpha(1-\beta)+\eta(1-\alpha)]}{\eta(1-\alpha)-\alpha \beta}, \\
g_{\chi}=-\frac{\theta-\beta}{\beta \theta} r+\chi-\frac{\rho}{\theta}, \\
g_{\psi}=g_{n}+\frac{\xi}{\delta}\left(B+g_{n}\right) \psi-\xi, \\
B=\frac{\xi[1-\beta-\eta(1-\alpha)]}{\eta(1-\alpha)} .
\end{gathered}
$$

To understand the dynamics of our system, two supplementary differential equations will be necessary, that is, the first equation of system (28) and

$$
g_{n}=\frac{\alpha \beta}{\eta(1-\alpha)-\alpha \beta} \chi-\frac{\alpha \xi}{\eta(1-\alpha)-\alpha \beta} .
$$

\section{Balanced Growth Equilibrium}

In this section, we focus our analysis on the balanced growth equilibrium, characterized by the fact that all variables grow at constant but possible different rates, and the shares of human capital in its different uses are constant. The system described above reaches the balanced growth path (BGP) if there exists $t_{*}$ (possibly infinite), such that for all $t \geq t_{*}$, $g_{u_{y}}=g_{u_{d}}=g_{u_{e}}=0$, and $g_{k}=g_{c}=g_{y}=g \neq g_{h}=g_{n}$, where $g_{z}$ denotes the growth rate of variable $z$. The following proposition gives our first main result that characterizes the balanced growth path.

Proposition 1. Let $\xi>\rho$ and $\theta>\theta_{M}$. Then the above system reaches the $B G P$ and the following statements are valid:

$$
\begin{gathered}
r_{*}=\frac{\theta \xi[\alpha(1-\beta)+\eta(1-\alpha)]-\eta \rho(1-\alpha)}{\theta[\alpha(1-\beta)+\eta(1-\alpha)]-\eta(1-\alpha)}, \\
\chi_{*}=\frac{(\theta-\beta) r_{*}+\beta \rho}{\beta \theta}, \quad \psi_{*}=\frac{\delta\left(\xi-g_{n_{*}}\right)}{\xi\left(B+g_{n_{*}}\right)}, \\
g_{n_{*}}=\frac{\alpha\left(\beta \chi_{*}-\xi\right)}{\eta(1-\alpha)-\alpha \beta}, \quad g_{*}=\frac{r_{*}-\rho}{\theta}, \\
u_{y_{*}}=\frac{(1-\beta-\eta)\left(\xi-g_{n_{*}}\right)}{\eta(1-\alpha)\left(B+g_{n_{*}}\right)}, \quad u_{e_{*}}=\frac{\alpha\left(\beta \chi_{*}-\xi\right)}{\xi[\eta(1-\alpha)-\alpha \beta]}, \\
u_{d_{*}=} \frac{\alpha\left(\xi-g_{n_{*}}\right)}{\xi\left(B+g_{n_{*}}\right)} \frac{\beta \chi_{*}-\xi}{\eta(1-\alpha)-\alpha \beta}, \quad u_{m_{*}}=\frac{\alpha \eta}{1-\beta-\eta} u_{y_{*}},
\end{gathered}
$$

where

$$
\begin{gathered}
\theta_{m}=\frac{\eta(1-\alpha)}{\alpha(1-\beta)+\eta(1-\alpha)}, \\
\theta_{M}=1-\frac{\rho}{\xi} \frac{\alpha(1-\beta)}{\alpha(1-\beta)+\eta(1-\alpha)} .
\end{gathered}
$$

Proof of Proposition 1. According to (30), constancy of $u_{y}$ implies that, at equilibrium, the growth rate of $n$ equals the growth rate of $h$; that is, $g_{n *}=g_{h *}$, and therefore $g_{\psi_{*}}=0$. Constancy of $g_{c_{*}}$ implies the constancy of $r_{*}$; that is, $g_{r_{*}}=0$. Therefore $g_{y_{*}}=g_{k_{*}}$, and thus $\chi$ is also constant in the steady state; that is, $g_{\chi_{*}}=0$ and therefore we have $g_{*}=g_{y_{*}}=$ $g_{k_{*}}$, where we denote by $g_{z *}$ the equilibrium's growth rate of variable $z$. From the second equation of (28) we observe that $r_{*}$ should be at least greater than $\xi$. Analyzing $r_{*}$ we conclude that the only acceptable alternative that ensures $r_{*}>\xi$ is $\theta>\theta_{m}$. This condition also ensures the positivity of $\chi_{*}$ if $\theta>\beta$. A simple computation procedure shows that $\eta(1-\alpha)-\alpha \beta>0$, that is, $\alpha<\alpha_{m}$, implies that $\beta \chi_{*}-\xi>0$ and $\eta(1-\alpha)-\alpha \beta<0$, that is, $\alpha>\alpha_{m}$, implies that $\beta \chi_{*}-\xi<0$ and therefore $g_{n_{*}}$ and $u_{e_{*}}$ are both positive. What we need now is to find conditions that ensure positivity of $\xi-g_{n_{*}}$. Obviously we have $\theta_{m}<\theta_{M}$. By direct computation we find that $\xi-g_{n_{*}}>0$ for all $\alpha>0$ and for all $\theta>\theta_{M}$. Because $u_{y_{*}}>0, u_{d_{*}}>0, u_{e_{*}}>0, u_{m_{*}}>0$ and $u_{y_{*}}+u_{d_{*}}+u_{e_{*}}+u_{m_{*}}=$ 1 we obviously have $\left\{u_{y_{*}}, u_{d_{*}}, u_{e_{*}}, u_{m_{*}}\right\} \in(0,1)$. The other relations follow immediately by direct computation and thus the proof is completed. 


\section{Stability Property of the BGP}

In this section we investigate the stability properties of the BGP found in the previous section. For our analysis we need to consider the system (37):

$$
\begin{gathered}
\dot{r}=\left[-\frac{(1-\beta)}{\beta} r+\frac{\eta(1-\alpha)}{\eta(1-\alpha)-\alpha \beta} \chi-A\right] r=f_{1}, \\
\dot{\chi}=\left[-\frac{\theta-\beta}{\beta \theta} r+\chi-\frac{\rho}{\theta}\right] \chi=f_{2}, \\
\dot{\psi}=\left[g_{n}+\frac{\xi}{\delta}\left(B+g_{n}\right) \psi-\xi\right] \psi=f_{3} .
\end{gathered}
$$

The study of stability equilibrium that we can examine here, that is, around the BGP, is called asymptotic stability or attracting and it describes how solutions that starts near the equilibrium point BGP converge to BGP. This is a local notion of stability. We will prove that the competitive equilibrium solution is locally unique; that is, the BGP is determinate, if the Jacobian of the reduced system has at least one eigenvalue with negative real part. There are two elements that are irrelevant for our analysis. We denote these two elements by $a$ and, respectively, by $b$. The Jacobian evaluated at BGP is given by

$$
J_{*}=\left(\begin{array}{ccc}
J_{11} & J_{12} & 0 \\
J_{21} & J_{22} & 0 \\
a & b & J_{33}
\end{array}\right),
$$

where

$$
\begin{gathered}
J_{11}=-\frac{(1-\beta)}{\beta} r_{*}, \quad J_{12}=\frac{\eta(1-\alpha)}{\eta(1-\alpha)-\alpha \beta} r_{*}, \\
J_{21}=-\frac{\theta-\beta}{\beta \theta} \chi_{*}, \\
J_{22}=\chi_{*}, \quad J_{33}=\xi-g_{n_{*}}>0 .
\end{gathered}
$$

Let $\omega_{1}, \omega_{2}, \omega_{3}$ be the three eigenvalues of Jacobian J. First observe that $\xi-g_{n_{*}}>0$ and therefore $\omega_{3}>0$; the third eigenvalue of $J_{*}$ is positive. The following proposition gives the necessary and sufficient conditions which ensure the local saddle-point stability of the BGP.

Proposition 2. Let $\xi>\rho$ and $\theta>\theta_{M}$. Then the following statements are valid.

(i) If $\alpha>\alpha_{m}$, then the BGP is a saddle-point equilibrium.

(ii) If $\alpha<\alpha_{m}$, then the BGP is an unstable equilibrium,

where $\alpha_{m}=\eta /(\eta+\beta)$.

Proof of Proposition 2. The matrix $J_{*}$ has as an immediate property, the fact that two of its eigenvalues are the eigenvalues of the matrix $J 1_{*}$ :

$$
J 1_{*}=\left(\begin{array}{ll}
J_{11} & J_{12} \\
J_{21} & J_{22}
\end{array}\right) .
$$

By direct computation we obtain

$$
\begin{aligned}
\operatorname{Det}\left(J 1_{*}\right)= & \frac{\theta[\alpha(1-\beta)+\eta(1-\alpha)]-\eta(1-\alpha)}{\theta[\eta(1-\alpha)-\alpha \beta]} r_{*} \chi_{*}, \\
\operatorname{Tr}\left(J 1_{*}\right)= & \theta \xi[\alpha(1-\beta)+\eta(1-\alpha)] \\
& -\xi[\alpha(1-\beta)+\eta(1-\alpha)]+\alpha \rho(1-\beta),
\end{aligned}
$$

where $\operatorname{Det}\left(J 1_{*}\right)$ is the determinant of $J 1_{*}$ and $\operatorname{Tr}\left(J 1_{*}\right)$ is the trace of $J 1_{*}$. First observe that $\operatorname{Tr}\left(J 1_{*}\right)>0$ for all $\theta>\theta_{M}$. Examining the signs of $\operatorname{Det}\left(J 1_{*}\right)$ we can claim the following.

(i) If $\alpha>\alpha_{m}$, then $\operatorname{Det}\left(J 1_{*}\right)<0$ and therefore the real part of $\omega_{1}$ is negative and the real part of $\omega_{2}$ is positive and $\left|\omega_{1}\right|<\omega_{2}$.

(ii) If $\alpha<\alpha_{m}$, then $\operatorname{Det}\left(J 1_{*}\right)>0$ and therefore the real part of $\omega_{1}$ is positive and the real part of $\omega_{2}$ is positive

and thus the proof is completed.

We conclude this section by noting that in our model there is not an upper limit for $\theta$ as it is the case of the cited paper of Manuel Gomez. Also we clarified some questionable aspects of the model developed by FSA and later analyzed by Manuel Gomez, Iacopetta, and Tiago Sequeira.

\section{Conclusions}

In this paper we explored the equilibrium dynamics of an innovative economy, via an endogenous growth model with physical capital, human capital, and R\&D. In the second section we developed a model of endogenous growth with innovation and derive the differential equations that describe the dynamics of the economy. We proved in the third section that, under general fairly conditions, the model reaches the balanced growth path and determined the values of all variables at BGP. Finally, the previous section was dedicated to investigate the stability properties of the BGP.

The main results proposed by our paper are given in Propositions 1 and 2. As we can observe, these results make some light on the general properties of the model.

We close this final section with some numerical simulations in order to confirm the theoretical aspects presented in our paper. As it was observed by Jorgenson and Fraumeni [10, page 17], analysing the US economy for the period 1973-1986, physical capital input is the most important source of growth and therefore $\eta<\beta$ seems to be evident. For our simulation procedure we consider the following benchmark values.

(a) $\beta=0.25, \eta=0.20, \xi=0.05, \delta=0.1, \rho=0.03, A=1$, $\theta=2$, and $\alpha=0.4$. This parametrization yields the following equilibrium:

$\theta_{m}=0.2857, \theta_{M}=0.5714, \alpha_{m}=0.444, r_{*}=0.0533$, $\chi_{*}=0.2017, \psi_{*}=0.3077, g_{*}=0.0117, g_{n_{*}}=0.0083$, $u_{y_{*}}=0.7051, u_{d_{*}}=0.0256, u_{e_{*}}=0.1667, u_{m_{*}}=$ $0.1026, \operatorname{Det}\left(J_{1}\right)=0.1936$, and $\operatorname{Tr}\left(J_{1}\right)=0.0417$ and the eigenvalues are $\omega_{1}=0.0208+0.4395 i, \omega_{2}=0.0208-$ $0.4395 i$, and $\omega_{3}=0.0417$. As we can observe, in this case, the equilibrium is unstable. 
(b) $\beta=0.25, \eta=0.20, \xi=0.05, \delta=0.1, \rho=0.03, A=1$, $\theta=2$, and $\alpha=0.5$. This parametrization yields the following equilibrium:

$\theta_{m}=0.2105, \theta_{M}=0.5263, \alpha_{m}=0.444, r_{*}=0.0524$, $\chi_{*}=0.1982, \psi_{*}=0.2467, g_{*}=0.0112, g_{n_{*}}=0.0088$, $u_{y_{*}}=0.6784, u_{d_{*}}=0.0218, u_{e_{*}}=0.1765, u_{m_{*}}=$ $0.1233, \operatorname{Det}\left(J_{1}\right)=-0.1764$, and $\operatorname{Tr}\left(J_{1}\right)=0.0412$ and the eigenvalues are $\omega_{1}=-0.3999, \omega_{2}=0.4411$, and $\omega_{3}=0.0412$. As we can observe, in this case, the equilibrium is saddle path stable.

\section{References}

[1] G. M. Grossman and E. Helpman, Innovation and Growth in the Global Economy, The MIT Press, Cambridge, Mass, USA, 1991.

[2] P. Romer, "Endogenous technological change," Journal of Economic History, vol. 98, no. 5, pp. 71-102, 1990.

[3] C. Eriksson, "Market failures in the R\&D growth model with endogenous labor supply," Journal of Public Economics, vol. 61, no. 3, pp. 445-454, 1996.

[4] M. Funke and H. Strulik, "On endogenous growth with physical capital, human capital and product variety," European Economic Review, vol. 44, no. 3, pp. 491-515, 2000.

[5] L. G. Arnold, "Endogenous growth with physical capital, human capital and product variety: a comment," European Economic Review, vol. 44, no. 8, pp. 1599-1605, 2000.

[6] M. A. Gómez, "Transitional dynamics in an endogenous growth model with physical capital, human capital and R and D," Studies in Nonlinear Dynamics and Econometrics, vol. 9, no. 1, article 5, 2005.

[7] T. N. Sequeira, "Transitional dynamics of an endogenous growth model with an erosion effect," Manchester School, vol. 76 , no. 4, pp. 436-452, 2008.

[8] M. Iacopetta, "Phases of economic development and the transitional dynamics of an innovation-education growth model," European Economic Review, vol. 54, no. 2, pp. 317-330, 2010.

[9] M. Iacopetta, "Formal education and public knowledge," Journal of Economic Dynamics and Control, vol. 35, no. 5, pp. 676693, 2011.

[10] D. W. Jorgenson and B. M. Fraumeni, "Education and productivity growth in a market economy," Atlantic Economic Journal, vol. 21, no. 2, pp. 1-25, 1993. 


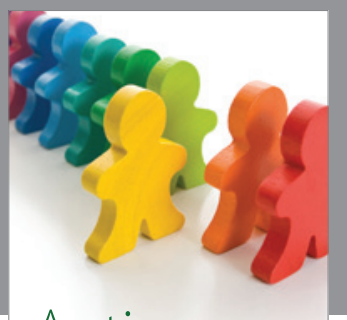

Autism

Research and Treatment
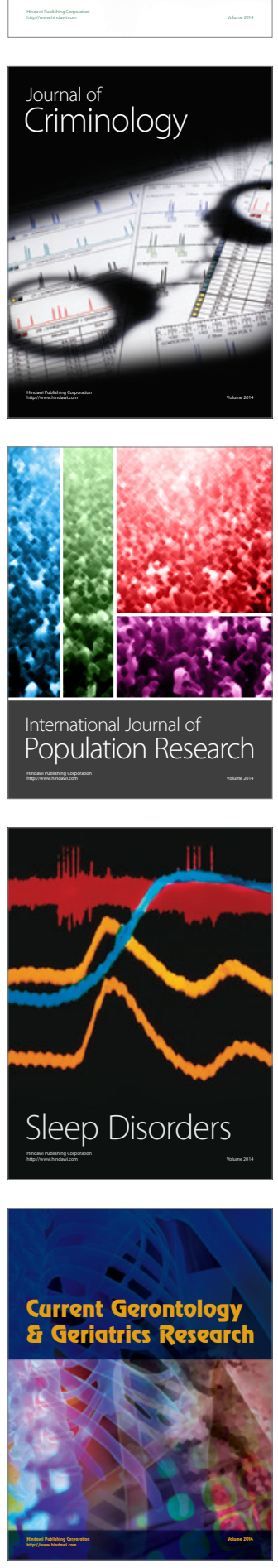
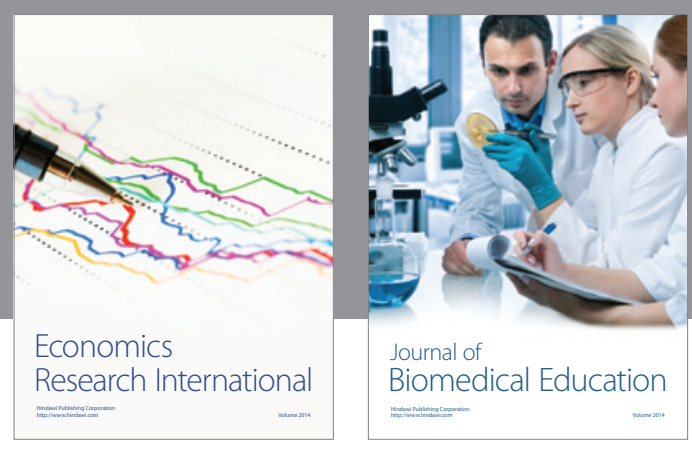

Journal of

Biomedical Education

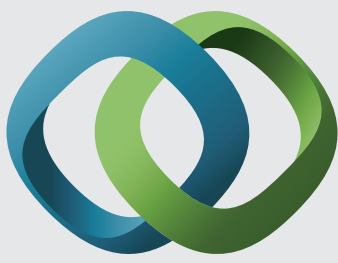

\section{Hindawi}

Submit your manuscripts at

http://www.hindawi.com
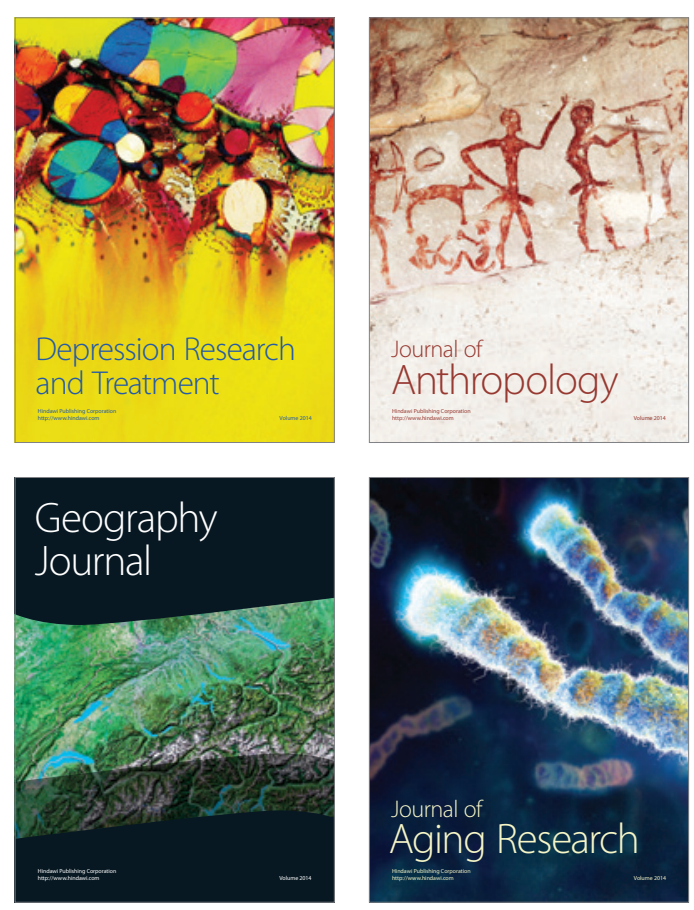

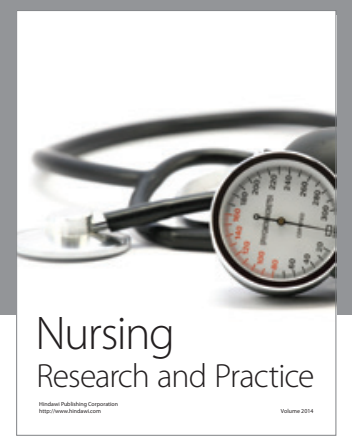

Nursing

Research and Practice

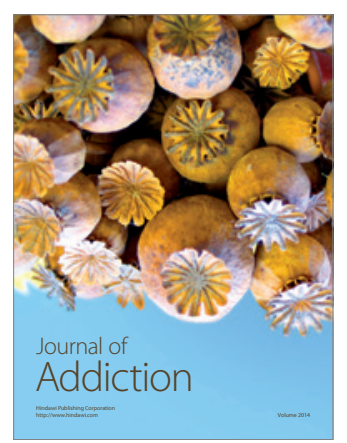

Child Development

Research

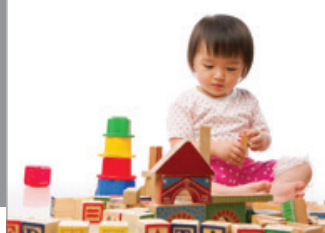

迥
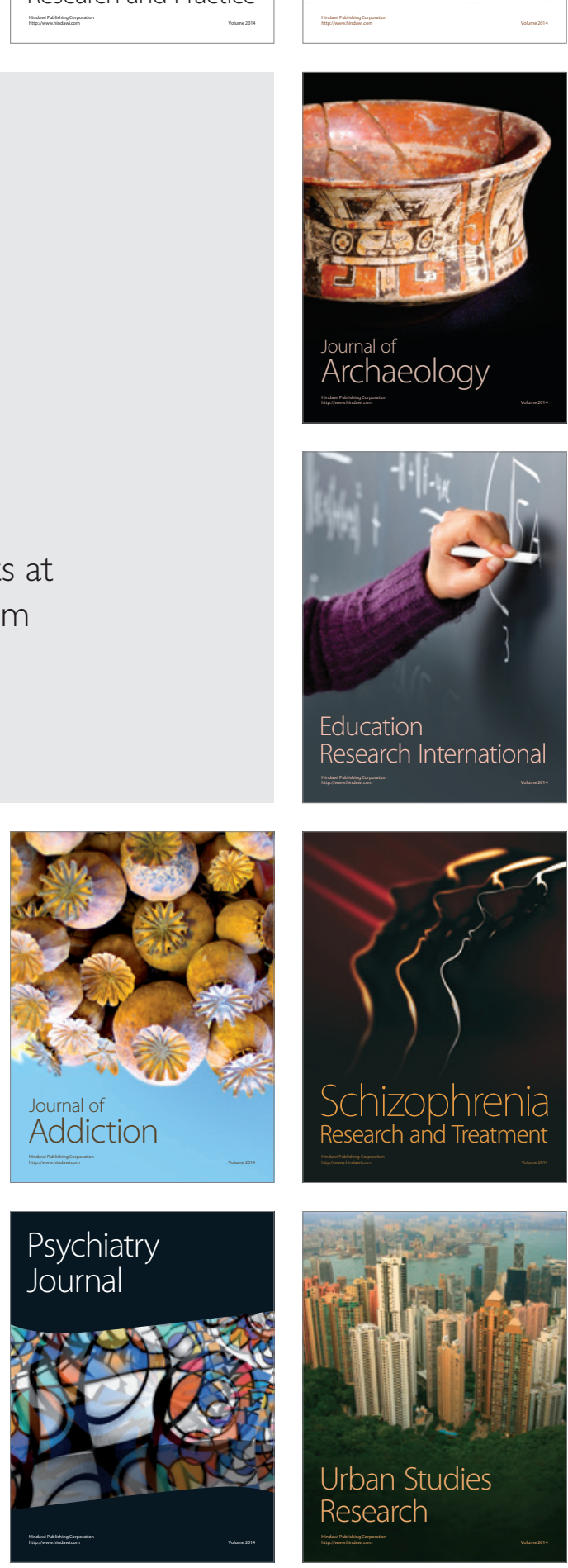